\title{
Radioguided Surgery of Parathyroid Adenomas and Recurrent Thyroid Cancer Using the "Low Sestamibi Dose" Protocol
}

\author{
Domenico Rubello, ${ }^{1 *}$ Lorraine M. Fig, ${ }^{2}$ Dario Casara, ${ }^{3 *}$ Andrea Piotto, ${ }^{4 *}$ Giuseppe Boni, ${ }^{5^{*}}$ \\ Maria R. Pelizzo, ${ }^{4 *}$ Brahm Shapiro, ${ }^{6}$ Sergio Sandrucci, ${ }^{7 *}$ Milton D. Gross, ${ }^{2}$ \\ and Giuliano Mariani ${ }^{*}$ \\ ${ }^{1}$ Nuclear Medicine Service, "S. Maria della Misericordia" Hospital, Rovigo, Italy \\ ${ }^{2}$ Nuclear Medicine Service, Department of Veterans Affairs Health System, Ann Arbor, MI \\ ${ }^{3}$ Nuclear Medicine Service, Azienda Ospedaliera di Padova, Padova, Italy \\ ${ }^{4}$ Department of Surgery, University of Padova Medical School, Padova, Italy \\ ${ }^{5}$ Regional Center of Nuclear Medicine, University of Pisa Medical School, Pisa, Italy \\ ${ }^{6}$ Division of Nuclear Medicine, Department of Radiology, University of Michigan Medical Center, \\ Ann Arbor, MI \\ ${ }^{7}$ Department of Surgery, University of Torino Medical School, Torino, Italy
}

\begin{abstract}
Purpose: The aim of this study was to establish the clinical efficacy of the "low sestamibi dose" (LSD) protocol to perform thyroid and parathyroid radioguided surgery in a large series of patients homogeneously studied and operated on by the same surgeon. The LSD protocol was initially developed in our center to cure primary hyperparathyroid (PHPT) patients with a high likelihood of a solitary parathyroid adenoma (PA) by minimally invasive radioguided surgery (MIRS). Since then, the same protocol has been applied to differentiated thyroid cancer (DTC) patients with ${ }^{131}$ I-negative, but sestamibi-positive, locoregional recurrent disease in order to obtain radical radioguided extirpation of tumoral lesions at reoperation.

Study Design: We reviewed the clinical charts of 453 consecutive patients referred at the surgical department at Padova University (Padova, Italy) to investigate a PHPT or a DTC recurrence: 336 patients (74.2\%) met the inclusion criteria for radioguided surgery, and these patients were analyzed for the aim of this study. There were 298 patients affected by PHPT with a high likelihood of a solitary sestamibipositive PA and 38 DTC patients affected by ${ }^{131}$ I-negative, but sestamibi-positive, locoregional recurrence. All patients underwent a preoperative imaging work-up, including sestamibi scintigraphy (doubletracer subtraction scan in PHPT patients and single-tracer, wash-out scan in DTC patients) and high-resolution neck ultrasonography (US). The LSD protocol we developed consists of the intravenous injection of a very low $(1 \mathrm{mCi})$ sestamibi dose in the operating theater just 10 minutes before commencing intervention for the purpose of radioguided surgery only. At variance with the traditional "high (20-25 $m C i)$ sestamibi dose (HSD)" protocol in which imaging and radioguided surgery are obtained in the same day, in the LSD protocol, imaging and radioguided surgery are performed in different days. The LSD protocol allows some advantages over the HSD protocol:
\end{abstract}

Address reprint requests to: Domenico Rubello; Nuclear Medicine Service_PET Unit, "S. Maria della Misericordia” Hospital, Istituto Oncologico Veneto (IOV), and GISCRIS; Viale Tre Martiti, 140, 45100 Rovigo, Italy; Tel.: +39 (0)425 394427; Fax: +39 (0)425 394434

E-mail: domenico.rubello@libero.it

*On behalf of the Italian Study Group on Radioguided Surgery and Immunoscintigraphy (GISCRIS). 
(1) more time for acquiring and interpreting preoperative imaging (planar scintigraphy, single-photon emission computed tomography [SPECT], US);

(2) an accurate selection of patients to whom MIRS is offered, especially in countries where the prevalence of nodular goiter with sestamibi-avid thyroid nodules (exclusion criteria for MIRS) is relatively high, as in mid-south-European countries;

(3) it facilitates the work planning in the operating theater (bilateral neck exploration requires an operating time of at least double to that of MIRS); and

(4) the radiation exposure dose to operating theater personnel is very low-substantially negligible, using the LSD protocol: This aspect assumes great importance in countries where radioproteximetric rules are stringent, as in Europe.

Results: PHPT patients. MIRS was successfully performed by a 1.5-2-cm skin incision in 287 of 298 PHPT patients (96.3\%) in whom such an approach was scheduled on the basis of preoperative imaging, including 41 of 57 patients (71.9\%) who had previously received thyroid or unsuccessful parathyroid surgery in another center. No case of major intraoperative complication was recorded. No case of persistent or recurrent PHPT was observed during postsurgical follow-up. DTC patients. A total of 79 metastatic lesions were intraoperatively detected by the gamma probe and successfully removed (68 of them had been correctly visualized at preoperative sestamibi scintigraphy). During subsequent follow-up, 18 patients (72\%) were considered disease-free, whereas 7 had persistent disease (increased serum thyroglobulin levels). The radiation exposure dose to the surgeon using the LSD protocol was 1.2 uSi/hour, that is, 20-30-fold lower than that delivered with the HSD protocol used for PHPT patients and with the

${ }^{131}$ I protocol used for DTC patients with recurrence.

Conclusions: On the basis of our data, it can be concluded that the LSD protocol is a safe and effective protocol to perform in both MIRS in PHPT patients and radioguided reoperation in DTC patients with ${ }^{131}$ I-negative recurrence. Furthermore, from a radioproteximetric point of view, in comparison with other radioguided protocols used for the same purposes, the LSD protocol minimizes the radiation-exposure dose to the surgeon and operating theater personnel.

Key words: radioguided surgery, primary hyperparathyroidism, parathyroid adenoma, differentiated thyroid carcinoma, recurrent disease, "low sestamibi dose" protocol.

\section{INTRODUCTION}

Recently, some new surgical approaches using intraoperative gamma probe and ${ }^{99 \mathrm{~m}} \mathrm{Tc}$-Sestamibi as radiotracer, have been proposed and optimized by several researchers and subsequently introduced in clinical practice

(1) to cure patients affected by primary hyperparathyroidism (PHPT) with a high preoperative likelihood of a solitary parathyroid adenoma (PA) and

(2) to cure patients affected by a limited locoregional ${ }^{131}$ I-negative, but sestamibi-positive, tumoral recurrence of differentiated thyroid carcinoma (DTC).

We have published our previous experience on minimally invasive radioguided surgery (MIRS) of $\mathrm{PA}^{1-5}$ and on radioguided surgery of
DTC patients with recurrence, ${ }^{6,7}$ thus, a fraction of the patients described in this study have just been reported in previous papers. The "low sestamibi dose" (LSD) protocol we developed for radioguided surgery is based on the administration to the patient of a very low $(1 \mathrm{mCi})$ sestamibi dose directly in the operating theater just a few minutes before commencing the intervention. Initially, we developed the LSD protocol in PHPT patients affected by $\mathrm{PA}^{1-5}$ and proposed it as an alternative to the "high (20-25) mCi sestamibi dose" (HSD) protocol first developed by Norman et al. ${ }^{8-10}$ and then used in other centers. ${ }^{11,12}$ Then, we validated the same LSD protocol for radioguided surgery of ${ }^{131}$ I-negative, but sestamibi-positive, locoregional recurrences in DTC patients ${ }^{6,7}$ as an alternative to the high $100-\mathrm{mCi}{ }^{131} \mathrm{I}$ dose protocol. ${ }^{13}$ In this regard, the main aims of this study were: 
(1) to verify the clinical feasibility and efficacy of the LSD radioguided protocol developed in our center, which is quite different from the other radioguided protocols traditionally used, and

(2) to demonstrate that the same LSD protocol can be used, both for PHPT patients with PA and for DTC patients with locoregional recurrence.

\section{MATERIALS AND METHODS}

The clinical records of a total of 453 consecutive patients referred to the Department of Surgery at Padova University (Padova, Italy) were reviewed for the aim of this study, which was conducted under the aegis of GISCRIS (Italian Study Group on Radioguided Surgery and Immunoscintigraphy).

\section{PHPT Patients}

A total of 400 consecutive patients affected by clinically and biochemically proven PHPT were studied and operated on by the same surgeon: 102 of them did not met the inclusion criteria for MIRS (48 of 102 had concurrent multinodular goiter with sestamibi-avid thyroid nodules, 36 of 102 had multigland parathyroid disease, of whom 4 were multiple endocrine neoplasia [MEN]-related, and 20 of 102 had a completely negative sestamibi scan) and were excluded from MIRS. Thus, for the aim of this study, only the group of 298 of the 400 PHPT patients (74.5\%) with a high probability of being affected by a solitary PA, on the basis of preoperative sestamibi imaging (see below) and in whom MIRS was offered, were considered. There were 246 females and 52 males, with a mean age of 54.4 (range, 18-84 years). It is worth noting that 57 patients had undergone previous thyroid or unsuccessful parathyroid surgery in another center.

We adopted the following inclusion criteria for offering MIRS:

(1) evidence at sestamibi scintigraphy of a solitary PA;

(2) a clear sestamibi uptake in the PA with a parathyroid-to-background $(\mathrm{P} / \mathrm{B})$ ratio higher than 1.2 ;

(3) absence at sestamibi scintigraphy and at high-resolution neck ultrasound (US) of coexisting thyroid nodules;
(4) no history of familial hyperparathyroidism or MEN; and

(5) no history of irradiation to the neck.

Preoperative imaging procedures included a single-session, double-tracer ${ }^{99 \mathrm{~m}} \mathrm{Tc}$-pertechnetate/ ${ }^{99 m}$ Tc-sestamibi subtraction scintigraphy and high-resolution neck US, as previously described. ${ }^{1-5}$ Briefly, the scintigraphic procedure included the following steps: at acquisition,

(1) patient was intravenously injected with 4 $\mathrm{mCi}$ of ${ }^{99 \mathrm{~m}} \mathrm{Tc}$-pertechnetate;

(2) 20 minutes later, the patient was invited to assume $400 \mathrm{mg}$ of potassium perchlorate $\left(\mathrm{KClO}_{4}\right)$ orally to speed the thyroid wash-out of $99 \mathrm{~m}$ Tc-pertechnetate (of note, as previously demonstrated, ${ }^{14}$ a 10 -minute time interval is necessary before $\mathrm{KClO}_{4}$ begins its action on thyroid ${ }^{99 m} \mathrm{Tc}$-pertechentate clearance);

(3) immediately after $\mathrm{KClO}_{4}$ administration, the patient was positioned under the gamma camera with the neck immobilized using an elastic band;

(4) a 5-minute ${ }^{99 \mathrm{~m}} \mathrm{Tc}$-pertechnetate thyroid image was acquired;

(5) soon after, without moving the patient, 15 $\mathrm{mCi}$ of sestamibi were intravenously injected, followed by a flush of saline to avoid radiotracer stagnation; and

(6) a dynamic acquisition of seven (5 minute each) sestamibi images was then acquired.

For processing,

(1) normalization of sestamibi images to the maximum pixel count activity of the pertechnetate image was obtained;

(2) movement correction on the $\mathrm{x}$ and $\mathrm{y}$ axis was done using an interactive program, when necessary; and

(3) subtraction of sestamibi images from the pertechnetate image was done using an interactive program with different subtraction thresholds. Scintigraphic images were stored in a $128 \times 128$ matrix and processed using a dedicated computer.

In patients with concordant sestamibi/US results (both positive or negative), no further imaging was obtained, whereas in cases with discrepant findings (sestamibi positive and US negative) a single-photon emission computed tomography 
(SPECT) examination ( $n=84$ patients) was also acquired to investigate a possible ectopic or deep position of the PA. SPECT was acquired just after the completion of planar sestamibi scintigraphy, thus using the same radiotracer dose; in this way, sestamibi reinjection was not necessary, as happens in some other protocols, ${ }^{11,12}$ thus avoiding additional radiation exposure to the patient and nuclear medicine service personnel.

All operations were performed by the same surgeon (M.R.P.) within 1-3 weeks from imaging. Postsurgical follow-up ranged from 20 to 83 months (mean \pm standard deviation $=38.4 \pm$ 8.2 months).

During postsurgical follow-up, all patients received clinical and laboratory (serum calcium and PTH levels) surveys 1 month after surgery and subsequently every 2-3 months thereafter.

\section{DTC Patients with Locoregional Recurrence}

A total of 53 DTC consecutive patients with locoregional recurrence occurred after previous total thyroidectomy and one or more ${ }^{131}$ I treatments were referred to the Department of Surgery at Padova University: 15 of them did not meet the inclusion criteria for radioguided surgery (see below) and were excluded. Thus, for the aim of this study, 38 patients $(71.7 \%)$ were enrolled for radioguided surgery. Inclusion criteria were:

(1) increase of serum thyroglobulin $(\mathrm{Tg})$ levels during follow-up (Tg levels were $101 \pm 192$ ng/dL on L-thyroxine therapy and $156 \pm 243$ ng/dL after hormonal withdrawal), despite ${ }^{131}$ I-negative whole-body scan;

(2) a clear positive sestamibi scan (lesion-tobackground (L/B) ratio higher than 1.2), showing the presence of locoregional recurrent disease in the neck; and

(3) absence of distant metastases established on the basis of clinical examination, whole-body computed tomography (CT) scan, wholebody bone scan, and whole-body 18-fluoro2-deoxyglucose-positron emission tomography (FDG-PET) scan.

Neck high-resolution US was also performed in the same session of scintigraphy, and the final diagnosis of recurrent disease was achieved by US-guided fine-needle aspiration cytology (USFNAC). They were 20 females and 18 males, with a mean age of $41.8 \pm 16.8$ years: At initial diagnosis and treatment, 30 patients had papillary histotype, 3 follicular, 2 Hurtle cells, 1 tall cells, and 2 with poorly differentiated insular pattern; TNM was pT4 in 27 cases, pT3 in 1, pT2 in 4, and pT1 in 6; metastatic lymph node metastases were found in 27 patients, whereas no patient had distant metastases.

In DTC patients with locoregional recurrence, preoperative imaging consisted of a dual-phase sestamibi scintigraphy. At variance with doubletracer pertechnetate/sestamibi subtraction scintigraphy performed in PHPT patients, in DTC patients with recurrent disease, pertechnetate scan was not performed owing to the absence of "normal" thyroid tissue as a consequence of previous total thyroidectomy and ${ }^{131}$ I ablative treatment. In detail, after the intravenous injection of $20 \mathrm{mCi}$ of sestamibi, 10 -minute early (20 minutes) and delayed ( 2 hours) planar images of the head and neck were obtained. Similarly to PHPT patients, also in the group of DTC patients with recurrent disease, imaging was stopped in the cases of congruent scintigraphic and US finding, whereas a SPECT imaging was also obtained in cases of incongruence ( 9 patients). SPECT imaging was acquired just after the early sestamibi planar acquisition. Neck US and US-FNAC were also performed in the same session of scintigraphy.

All patients were operated on by the same surgeon (M.R.P.) within 1-3 weeks from imaging. During a traditional bilateral neck exploration (BNE), the gamma probe was intraoperatively used to detect the sestamibi-avid tumoral foci shown at preoperative scintigraphy.

Postsurgical follow-up ranged from 11 to 58 months (mean \pm standard deviation $=17.4 \pm 7.6$ months). All patients underwent detailed investigation (at 1 and 2 months and then at 6-12-month intervals) by means of clinical examination, serum $\mathrm{Tg}$ and anti-Tg antibody measurements, neck US, and other imaging modalities, as necessary.

Both for PHPT and DTC patients with recurrence, planar scintigraphic imaging was acquired by a large-field-of-view (LFOV) gamma camera (Orbiter, 7500; Siemens, or E-CAM, Siemens) equipped with a parallel-hole, low-energy, highresolution collimator. Images were stored in a $128 \times 128$ matrix and processed using a dedicated computer. SPECT was performed by a dual-head gamma camera (Axis, Picker International [Cleveland, $\mathrm{OH}$ ], or E-CAM, Siemens [Hoffman Estates, IL]) equipped with a parallelhole, low-energy, high-resolution collimator adopting the following parameters: for acquisition, elliptical orbit, $60 \times 2$ steps, 30 seconds per 
step, $128 \times 128$ matrix; for reconstruction, a Batterworth filter (Picker International and Siemens) was used, with a cut-off ranging from 0.4 to 0.65 and an order ranging from 5.0 to 6.0 , using a dedicated computer: Three-dimensional (3D) analysis was also obtained. Scintigraphic images were interpreted by three skilled nuclear medicine physicians; in cases of discrepancy, final diagnosis was reached by consensus.

Neck US was performed by a high-resolution 10$\mathrm{MHz}$ transducer (Technos; Esaote, Italy). Longitudinal and axial neck scans were obtained from the angle of the mandible to the sternal notch.

Steps of intraoperative LSD radioguided protocol developed in our center and used both for PHPT and DTC patients are resumed in Table 1.

For radioguided surgery, a hand-held commercially available collimated gamma probe (Scintiprobe MR 100; Pol.hi.tech., Carsoli [AQ], Italy) was used. The probe's characteristics were: linear collimated probe with an external diameter of $11 \mathrm{~mm}$, mounting a NaI scintillation detector; lateral shielding efficiency of $99 \%$ for $99 \mathrm{~m}_{\text {tech- }}$ netium; energy range of 30-385 KeV; gamma- detecting efficiency of $99 \%$ for $99 \mathrm{~m}_{\text {technetium }}$ peak energy $(140 \mathrm{KeV})$; and sensitivity threshold of $10 \mathrm{nCi}$ and spatial resolution of $5 \mathrm{~mm}$ at a 2$\mathrm{mm}$ distance.

The 1-mCi dose of sestamibi, for the purpose of radioguided surgery, was injected to the patient in the operating theater by the nuclear medicine physician, who then assisted the surgeon during intervention.

In patients with PHPT, intraoperative QPTH was also measured by imunochemoluminescent assay (Liason; Byk Gulden, Milan, Italy). A fall of at least 50\% in PTH levels 10 minutes after PA removal, in comparison with baseline preexcision value, was considered indicative of a successful parathyroidectomy. Additional blood samples for QPTH measurement were obtained in patients with multigland disease and after removal of any surgical specimen suspected of being hyperfunctioning parathyroid tissue.

Data were expressed as the mean \pm 1 standard deviation. Mean values were compared using the Student's $t$ test. P-values lower than 0.05 were considered significant.

Table 1. Procedural Steps to Perform Radioguided Surgery Using the Low Sestamibi Dose (LSD) in Patients with Solitary PA or Locoregional Recurrence of DTC

(a) In PHPT patient, blood samples are drawn from a peripheral vein both before commencing surgery and 10 minutes following PA removal to measure intraoperative QPTH levels.

(b) $1 \mathrm{mCi}$ of sestamibi is injected in the operating room 10 minutes before the start of surgery.

(c) Prior to surgical incision, patient's neck is scanned with a 11-mm collimated probe to individualize the maximum count activity area corresponding to the cutaneous projection of the PA or the DTC recurrence.

(d) In PHPT patients, a transverse 1.5- to 2-cm midline neck access (approximately $1 \mathrm{~cm}$ above the sternal notch) is performed (with this approach, a conversion to a BNE is easily obtainable, if necessary, in PHPT patients).

In DTC patients, a collar incision is performed. The gamma probe is used during a traditional BNE to intraoperatively detect the sestamibi-avid metastatic foci.

(e) A 11-mm collimate probe is repeatedly inserted through the small skin incision in PHPT patients (or through the collar skin incision in DTC patients), guiding the surgeon to the maximum count activity area corresponding to the PA or the DTC recurrence.

In some PHPT patients with a PA located deep in the neck, the ligature of middle thyroid vein and of inferior thyroid artery is required.

(f) Radioactivity is measured with the probe on the PA (or on DTC recurrence), thyroid gland (in PHPT patient), and background.

(g) Radioactivity is measured on the ex vivo PA (or on DTC recurrence) to evaluate successful removal of parathyroid (or thyroid neoplastic) tissue.

(h) Radioactivity is checked on the empty operating basin to evaluate completeness of parathyroid (or DTC recurrence) removal.

(i) Tissue ratios are calculated.

QPTH, quick parathyroid hormone; BNE, bilateral neck exploration; PA, parathyroid adenoma; DTC, differentiated thyroid carcinoma. 


\section{RESULTS}

\section{PHPT Patients}

Two hundred ninety-eight (298) consecutive patients with a high preoperative probability of being affected by a solitary PA were offered with MIRS. It is worth noting that whereas Sestamibi scintigraphy revealed a solitary PA in all 298 patients (a sestamibi positivity being the main inclusion criteria for MIRS), high-resolution US revealed a PA in 214 (US sensitivity $=71.8 \%$ ). MIRS was successfully performed in 287 of 298 patients $(96.3 \%)$ including the majority of patients who have received previous thyroid or parathyroid surgery (41 of 57 patients; 71.9\%).

In 11 patients, a conversion to BNE was required; in 2 patients because of intraoperative diagnosis at frozen-section analysis of a parathyroid carcinoma; in 4 because of persistently high QPTH levels after removal of the preoperatively visualized PA (in these 4 cases, a second PA was found at subsequent neck exploration); and in 4 because of a difficult-to-remove PA owing to hard position and/or big size of the gland. It is worth noting that in these 11 patients, preoperative SPECT examination was not obtained because of the positivity and agreement of planar sestamibi scintigraphy and high-resolution neck US results for the presence of a solitary PA.

In patients who were successfully treated by MIRS, the gamma probe was judged by the surgeon to be particularly useful for the detection of 23 ectopic PAs located in the mediastinum, 2 ectopic PAs located at the carotid bifurcation, and 21 PAs located deep in the neck in the pararetroesophageal/para-retrotracheal spaces. From a cosmetic point of view, a small 1.5-2-cm skin incision was sufficient to perform MIRS. The mean operating time was 33.4 minutes (range, 13-60), and the mean hospital stay was 1.1 days (range, 1-2 days). Local anesthesia was successfully performed in 75 patients, the majority of whom $(n=62)$ were elderly patients with concomitant invalidating diseases, thus contraindicating general anesthesia. No major surgical complication (laryngeal nerve palsy, permanent hypoparathyroidism) was recorded. Transient hypocalcemia was observed in $9.4 \%$ of cases. No case of persistent or recurrent PHPT was observed during subsequent follow-up.

Concerning the outcome of the 11 patients in whom a conversion to BNE was required, no major intraoperative complications (permanent hypoparathyroidism or larynx nerve palsy) was ob- served; the 2 patients with parathyroid carcinoma were eucalcemic after intervention and did not experience recurrence (at present, the follow-up is 62 and 43 months, respectively); a transient hypocalcemia was observed in 5 of 11 patients (45.4\%), of whom 2 were with parathyroid carcinoma and 3 with double PA.

Concerning the group of PHPT patients in whom SPECT was performed, it is worth noting that in 27 patients, the SPECT analysis correctly suggested a deep location of PA, which was then confirmed at surgery.

The $\mathrm{P} / \mathrm{B}$ ratio was $2.63 \pm 1.12$, and the counts in the empty parathyroid bed after PA removal were $0.9 \pm 0.1$. The weight of the excised PA removed by MIRS was $913 \pm 292 \mathrm{mg}$. In the 2 cases of parathyroid carcinoma, the weight was 750 and $1140 \mathrm{mg}$, respectively.

The radiation-exposure dose to the surgeon using the LSD protocol was $1.2 \mathrm{uSi} /$ hour.

\section{DTC Patients with Locoregional Recurrence}

A total of 79 sestamibi-positive metastatic lesions were removed under gamma-probe guidance. It is worth noting that 68 of these lesions had been visualized at preoperative sestamibi scintigraphy: The higher number of sestamibi-positive lesions $(n=79)$ found intraoperatively can be explained by the fact that some of the hot spots defined as "single" at preoperative sestamibi scintigraphy were found to be constituted by "multiple" adjacent metastatic lesions at radioguided surgery. In detail, we removed (1) 56 metastatic lymph nodes, of which 41 were from the central compartment and 15 from the laterocervical compartment, and 23 recurrences in the thyroid bed; and (2) The mean size of the metastatic lesions was $18.7 \pm 8.3 \mathrm{~mm}$, with a range of $5-40 \mathrm{~mm}$. The lesion-to-background ratio was $2.44 \pm 0.93$, and the counts in the empty surgical basin after recurrence removal was $0.9 \pm 0.1$.

It is interesting to point out that at preoperative scintigraphic imaging, all the 68 sestamibipositive lesions were clearly visualized in the early (20 minutes) images, whereas only 54 of them remained clearly depicted in the delayed ( 2 hours) images: This can be explained by a relatively rapid sestamibi wash-out from some DTC metastatic lesions.

Concerning the small group of DTC patients in whom SPECT was performed, it is worth noting that in 2 of them, SPECT analysis correctly suggested a deep location of the tumoral lesion, which was then confirmed at surgery. 
Neck US identified at least 1 metastatic lesion in all patients, and in the whole series, US identified 43 metastatic lesions: In these lesions, USguided FNAC was performed to confirm diagnosis of malignancy. Thus, the overall sensitivity of US was $54.4 \%$ and that of preoperative Sestamibi was $86.1 \%$.

The probe was judged by the surgeon of particular usefulness in patients with lymph nodal metastases located outside the thyroid bed and the central compartment (i.e., those located in the laterocervical compartment), as well as to check the empty surgical basin after lesion removal to verify successful extirpation of the tumor.

After a postreoperation follow-up of $17.4 \pm$ 7.6 months, 18 patients $(72 \%)$ were considered disease-free, whereas 7 had persistent metastatic disease (increased serum $\mathrm{Tg}$ levels).

The radiation exposure dose to the surgeon using the LSD protocol was $1.2 \mathrm{uSi} /$ hour, that is, 25 -30-folds less than protocols using ${ }^{131} \mathrm{I}^{11}$

\section{DISCUSSION}

Radioguided surgery has gained a relevant clinical role over the last 15 years, both in PHPT patients with a high probability of a solitary $\mathrm{PA}^{1-11}$ in alternative to $\mathrm{BNE}^{15}$ and in DTC patients with locoregional disease recurrence after total thyroidectomy and ${ }^{131}$ I therapy. ${ }^{6,7,16}$ The objects of radioguided surgery in these 2 pathologies is quite different: In PHPT patients with a solitary PA, the main object is to remove the PA through a small skin incision, thus to perform MIRS, whereas in DTC patients with ${ }^{131}$ I-negative locoregional recurrence, the main object is to improve the radicality of surgery at reoperation. The first developed protocols for radioguided surgery are the HSD protocol for PHPT patients ${ }^{8-10}$ and the ${ }^{131}$ I protocol for DTC patients with locoregional recurrence. ${ }^{13}$

Concerning PHPT patients, the moving of surgical strategy towards minimally invasive approaches was mainly related to

(1) the consciousness that PHPT is a result of a solitary PA in the majority of PHPT patients (approximately 85\%-90\% of cases) and

(2) the technical improvements available in surgical practice, such as the microsurgery instruments, endoscope, peroperative measurements of QPTH, and intraoperative gamma probe.
Presently, more than half of all endocrine surgeons are using approaches of minimally invasive parathyroidiectomy, performing the removal of a solitary PA through a small midline or lateral neck skin incision of $1-2 \mathrm{~cm} .{ }^{17}$ Obviously, in contrast to BNE, when considering a minimally invasive parathyroidectomy, an accurate preoperative imaging is mandatory mainly

(1) to establish whether the PA is really solitary or not and

(2) to obtain a precise localization of the PA.

Specifically, considering MIRS—at variance with BNE-MIRS requires stringent inclusion criteria, mainly related to the sensitivity and accuracy of sestamibi scintigraphy ${ }^{18-23}$ :

(1) a high likelihood of a solitary PA demonstrated by preoperative sestamibi imaging;

(2) a clear sestamibi uptake in the PA; and

(3) absence of concomitant sestamibi-avid thyroid nodules.

In agreement with other reported studies on MIRS, ${ }^{1-12}$ adopting the selection criteria cited above, in our series, approximately $70 \%$ of PHPT patients were offered with MIRS. Thus, the success of MIRS strictly depends upon a precise preoperative localization of a solitary PA. In this regard, there is evidence in the literature that the combination of sestamibi scintigraphy and US can provide important advantages such as:

(1) more accurate information about PA localization (site, deepness);

(2) differentiation of solitary PA from multigland disease; and

(3) evaluation of the presence of coexisting nodular goiter. ${ }^{1-24}$

There is also evidence in the literature that the completion of sestamibi planar scintigraphyperformed both with the dual-phase or doubletracer subtraction technique-with SPECT acquisition improves sensitivity and accuracy of the imaging procedure, especially providing information (1) on the deepness of the PA; (2) on the presence of multigland disease; and (3) on a more accurate P/B ratio calculation. ${ }^{24-27}$ Furthermore, some authors reported a higher sensitivity using the pinhole SPECT than with the parallel-hole SPECT $^{28,29}$ and with the SPECT/CT fusion imaging. ${ }^{30}$ 
The first MIRS protocol for PHPT patients was developed by Norman in $1997 .{ }^{8-10}$ It consists of a single-day imaging and surgery approach. The patient is injected with a $740-925-\mathrm{MBq}$ dose of sestamibi, and scintigraphic images are obtained by dual-phase technique, then MIRS is performed within 2-3 hours from radiotracer administration through a lateral neck access. A further delay in performing MIRS can cause false-negative results at radioguided surgery owing to a relatively rapid sestamibi wash-out from some PA; this condition should be discouraged. ${ }^{24}$ Norman's protocol is very attractive from a cost-analysis perspective, because sestamibi scintigraphy and MIRS are performed on a same-day basis and a single radiotracer dose is used both for imaging and surgery. However, Norman's protocol also presents some practical disadvantages, given the uncertainty of the scintigraphic results and the differences between the MIRS and BNE with respect to the need for operating theater planned time (at least double with BNE to that with MIRS) and efficient patient scheduling. This problem would be expected to be particularly relevant in areas with a high prevalence of nodular goiter, so that a different-day protocol-as the protocol we used-should be preferable. ${ }^{1-5,24}$ The protocol we developed is a different-day protocol. ${ }^{1-5}$ On the first day, localizing imaging is obtained by means of a double-tracer scintigraphy combined with neck US. On the day of MIRS, a low (1 mCi) sestamibi dose is given directly in the operating theater a few minutes before surgery by the nuclear medicine physician. Our so-called "low sestamibi dose" protocol provides some advantages over Norman's HSD protocol as:

(1) A very low-negligible radiation exposure dose is delivered to the patient and operating theatre personnel (this aspect is particularly desiderable in countries were proteximetric rules are very stringent, as in Europe); and

(2) Potential false-negative results related to PA characterized by rapid sestamibi wash-out can be disclosed.

At variance with the HSD protocol in our LSD protocol, we prefer to perform MIRS though a midline neck access because a conversion to BNE is easier, when necessary. ${ }^{31}$ As demonstrated in this study, the clinical efficacy and safety of the LSD protocol is substantially equal to that of the HSD protocol, ${ }^{8,9}$ with a success rate higher than
95\% and without major intraoperative complications. Furthermore, as with the HSD protocol, ${ }^{10}$ in this study, using the LSD protocol, we were also able to perform MIRS in the majority of patients who had previously undergone a thyroid/parathyroid surgery, as well as demonstrating that in using the LSD protocol, MIRS can be performed under local anesthesia with an early (same-day) hospital discharge similar to that of HSD protocol..$^{8-10}$ It is worth noting that the LSD protocol was judged by the surgeon to be particularly useful in detecting PA located in an ectopic or deep site. Moreover, it has to be pointed out that both the Norman's HDS protocol and our LSD protocol are actually used in other surgical centers. ${ }^{1,12,24,32}$ It appears reasonable to think that Norman's single-day HSD protocol can be preferable in patients with a low likelihood of nodular goiter, whereass our different-day LSD protocol should be preferred in areas with a high prevalence of nodular goiter. Interestingly, using the HSD protocol in a large series of 345 hyperparathyroid patients, Murphy and Norman ${ }^{9}$ found that any surgical specimen counting more than $20 \%$ of the background activity was invariably associated with a solitary PA: This was named "the $20 \%$ rule." In this light, a $100 \%$ accuracy was calculated by the authors for the differential diagnosis of a solitary PA versus a multigland disease, thus making it unnecessary to perform QPTH. ${ }^{9}$ Unfortunately, this rule doesn't work with our LSD protocol; in particular, in all 4 cases with a double PA diagnosed intraoperatively, the PA-to-background percentage of the first-removed PA was higher than 20\%-and in these patients, a conversion to BNE was strongly suggested by the persistence of high QPTH levels. Thus, it appears that the $20 \%$ rule cannot be applied to the LSD protocol, in which the measurement of QPTH is strongly recommended. This discrepancy could be explained by the fact that the sestamibi-kinetic and, consequently, the tissue ratios, are different when intraoperative measurements are obtained within 30-40 minutes from injection, as happens in the LSD protocol, in comparison to measurements obtained 2-3 hours after sestamibi injection, as happens with the HSD protocol.

Concerning the DTC patients with locoregional recurrence, ${ }^{131} \mathrm{I}$ is the main radiotracer used in the treatment of patients with recurrent DTC. However, although ${ }^{131} \mathrm{I}$ is characterized by very high specificity (99\%-100\%), only a fraction of DTC recurrences are ${ }^{131}$ I-avid (50\%-60\% of pap- 
illary and $64 \%-67 \%$ of follicular cancers). ${ }^{33}$ The loss of capability to trap ${ }^{131}$ I by metastatic disease determines limitations, both for a diagnostic and therapeutic point of view (iodine-negative: so-called nonfunctioning metastases). ${ }^{34}$ Nonfunctioning metastases can be diagnosed at all ages, but their prevalence increases in the elderly, in poorly differentiated histotypes, and in tumors revealing an aggressive pattern. ${ }^{34}$ In these patients, an accurate localization of nonfunctioning metastases at an early stage (i.e., when they are spreaded only to the neck and mediastinum) can be crucial because only a prompt radical reoperation can offer an improvement in the patient's outcome and survival. ${ }^{35,36}$ Radiological imaging modalities, such as CT scan and MRI, have shown a relatively low sensitivity in detecting locoregional metastases of DTC. ${ }^{36}$ In contrast, high-resolution neck US and some tumorseeking scintigraphic methods, such as sestamibi scan, have proven high sensitivity in revealing cervical and mediastinal DTC recurrences. ${ }^{34-36}$ In these patients, radioguided surgery using ${ }^{131} \mathrm{I}$ has demonstrated to be effective. ${ }^{13}$ The major drawback of the ${ }^{131}$ I protocol is related to the relatively high radiation-exposure dose delivered to the surgeon and operating theater personnel, approximately 20-30 times higher than the LSD protocol used for PHPT patients. ${ }^{13}$ Boz et al. ${ }^{16}$ proposed radioguided surgery of DTC patients with tumor recurrence after sestamibi injection with the HSD protocol. The authors decribed the case of a 30-year-old woman who previously underwent two operations and ${ }^{131}$ I ablative treatment for a well-differentiated follicular thyroid carcinoma. Approximately 7 years after initial treatment, a diagnosis of nonfunctioning, nonpalpable neck recurrence was made on the basis of a progressive increase of serum $\mathrm{Tg}$ levels and a positive sestamibi scan showing a neck focal radiotracer uptake. In this patient, radioguided surgery was performed 2 hours after the intravenous injection of $20 \mathrm{mCi}$ of sestamibi. The tumor-to-background ratio in the tumoral mass was high (4:1), and the recurrence embedded in the scar tissue was successfully removed. Complete tumor resection was confirmed by a subsequent sestamibi scan control, and serum Tg levels decreased to undetectable values during subsequent follow-up. In another feasibility study, Rubello et al. $^{6}$ studied 8 consecutive patients previously treated by total thyroidectomy and 1 or more ${ }^{131} \mathrm{I}$ treatments who developed, during subsequent follow-up, recurrent locoregional nonfunctioning recurrences visualized at sestamibi scan. At variance with Boz et al., ${ }^{16}$ the radioguided procedure used by Rubello et al. ${ }^{6}$ consisted of the intravenous injection-in the operating theater and a few minutes before the beginning of the intervention - of a very low $(1 \mathrm{mCi})$ sestamibi dose (i.e., the LSD protocol), followed by a flush of saline $(30 \mathrm{~mL})$ to avoid tracer stagnation. The authors chose to inject the radiotracer in the operating theater a few minutes before the beginning of the intervention, because it has been reported that DTC metastases can be characterized by a relatively rapid sestamibi wash-out, thus in order to avoid possible false-negative intraoperative results. ${ }^{6}$ Moreover, the injection of the radiotracer just before the beginning of surgery allowed for the authors to administer a very low sestamibi activity, 20-fold lower than that used in the HSD protocol of Boz et al., ${ }^{16}$ thus minimizing the radiation-exposure dose of the surgeon and operating theater personnel. The steps of radioguided surgery with the LSD protocol in DTC patients with locoregional recurrent disease are resumed in Table 1. In 7 of 8 patients, the radioactivity measured in the tumoral bed after lesion extirpation fell to background activity values, and, in 6 patients, a stable normalization of serum $\mathrm{Tg}$ was achieved after reoperation, suggesting a complete cure: In the other patient, a persistent slight increase in serum $\mathrm{Tg}$ levels was observed after surgery, despite the absence of pathological uptake in the neck and the absence of distant metastases, as evaluated using CT, MRI, and Sestamibi scans. This patient was judged as having a "biochemically" persistent disease. In the 8th patient, the persistence of gamma probe-detected high radioactivity levels in the tumoral bed after lesion removal correctly suggested disease persistence (deep tracheal infiltration), leading us to treat the patient with additional external radiotherapy. The authors did not find a significant correlation between tumor size and tumor sestamibi uptake; however, it is worth noting that, in most patients, a prompt fall in gamma probe-detected radioactivity in the neck after tumor removal greatly helped the surgeon to evaluate the completeness of extirpation. ${ }^{6}$ The data of this study, obtained in a large patient population, confirm the preliminary results reported by the same authors. ${ }^{6}$ Of course, to plan a radioguided surgery for DTC patients with recurrent disease, it is necessary that the metastatic deposits are capable of trapping sestamibi: In this study, we encountered this condition in approximately $70 \%$ of cases. Moreover, 
it is worth noting that the surgeon judged the LSD protocol to be of particular usefulness in patients with recurrence located in the lymph node of the laterocervical compartment and to check the empty surgical basin after lesion extirpation to confirm the completeness of the intervention.

An important topic to be discussed regards the use of sestamibi SPECT acquisition other than planar scintigraphic imaging. In our preoperative imaging work-up, we acquired SPECT only in patients with a discrepancy between planar scintigraphy and US examination. In this way, we found useful the performance of SPECT in revealing a PA or a DTC recurrence located deep in the neck: This information helped the surgeon in planning the intervention. However, there are some other aspects which need to be underlined:

(1) Some other authors recommended to systematically perform sestamibi SPECT acquisition ${ }^{25-29}$ because they found an increase in sensitivity of SPECT in comparison with planar sestamibi scintigraphy;

(2) There is also evidence in the literature that some acquisition protocols using the pinhole SPECT are characterized by a higher spatial resolution, in comparison with the parallelhole SPECT ${ }^{28,29}$ : This is highly desiderable, especially for detection of small PA or small DTC recurrences;

(3) With the currently available multihead gamma cameras, the time required to obtain a SPECT acquisition is relatively short (usually no more than 20-30 minutes).

Another important innovation in preoperative imaging is related to the recent availability of hybrid SPECT/CT gamma cameras. Some preliminary results suggest a higher accuracy of SPECT/CT systems over SPECT alone in a precise localization of PA. ${ }^{30}$ Further studies are necessary to confirm these promising results. Furthermore, concerning the imaging work-up of DTC patients with locoregional recurrence, there are studies in the literature that demonstrated a high sensitivity of the FDG-PET ${ }^{37-39}$; moreover, some preliminary experiences about the use of an intraoperative probe with FDG have provided promising results.

The second topic that needs to be discussed regards the kinetic of sestamibi, and in particular, its retention into the PA or the DTC recurrence. There is evidence in the literature that in both these diseases, the sestamibi wash-out can be rel- atively rapid, ${ }^{24,35}$ so that the traditional delayed (2-3 hours) sestamibi images can be negative, as observed in several patients in this study. To this regard, some authors reported higher sensitivity of early sestamibi images, both in PHPT patients $^{24,27}$ and in DTC recurrences. ${ }^{35}$ This observation can have some implication in radioguided surgery, too. In the LSD protocol we proposed, the sestamibi dose is injected in the operating theater just 10 minutes before radioguided surgery without waiting 2-3 hours, as scheduled in the traditional HSD Norman's protocol.

A third topic to be discussed regards the costbenefit analysis. The traditional single-day HSD protocol of Norman is very attractive because the same radiotracer dose is used for imaging and radioguided surgery. However, in using the different-day LSD protocol developed by us, it has to be emphasized that sestamibi is often used in a nuclear medicine service on a daily basis to perform myocardial perfusion scintigraphy, parathyroid scintigraphy, scintimammography, and other tumor-seeking scintigraphies (each of these examinations require approximately $15-25 \mathrm{mCi}$ ): in light of this, the cost of a 1-mCi sestamibi dose for the purpose of radioguided surgery is substantially negligible. Lastly, in this study, highresolution neck US showed a lower sensitivity, both in detecting PA in PHPT patients and recurrent disease in DTC patients. However, US was very useful in PHPT patients

(1) to diagnose concomitant multinodular goiter;

(2) to differentiate a PA located behind the thyroid gland shape from a thyroid nodule;

(3) to confirm the sestamibi scintigraphy finding of multigland parathyroid disease;

(4) to decide, in our protocol, patients to whom to perform a SPECT acquisition other then a planar scintigraphy (incongruent data, positive planar scintigraphy, and negative US examination); and

(5) in DTC patients with recurrent disease, US was useful to guide the operator to perform the FNAC.

\section{CONCLUSIONS}

In conclusion, on the basis of the data from this study, based on a large patient population, the LSD protocol we developed appears to be a safe and effective protocol for the purpose of radioguided surgery, both in PHPT patients with a high 
preoperative likelihood of being affected by a solitary PA and in DTC patients with ${ }^{131}$ I-negative, but sestamibi-positive, locoregional recurrence. At variance with the traditional HSD sameday protocol of Norman, the different-day LSD protocol developed in our center allows

(1) to plan the operating theater workflow better and

(2) to deliver to the surgeon and the operating theater personnel a low-negligible radiation exposure dose.

This last aspect is particularly appreciated in countries where the regulatory laws on radioproteximetry are very stringent, as in the Europe. Moreover, the injection of sestamibi in the operating theater just a few minutes before commencing the radioguided intervention can avoid possible false-negative results related to a rapid wash-out of sestamibi from the PA or DTC recurrence. Lastly, for the purpose of an accurate selection of patients candidable to sestamibi radioguided surgery, it seems reasonable to recommend the routine performance of SPECT acquisition other than planar scintigraphy.

\section{REFERENCES}

1. Casara D, Rubello D, Piotto A, et al. ${ }^{99 m}$ Tc-MIBI radioguided minimally invasive parathyroid surgery planned on the basis of a preoperative combined ${ }^{99 \mathrm{~m}} \mathrm{Tc}-$ pertechnetate $/{ }^{99 \mathrm{~m}} \mathrm{Tc}-\mathrm{MIBI}$ and ultrasound imaging protocol. Eur J Nucl Med 2000;27:1300.

2. Casara D, Rubello D, Pelizzo MR, et al. Clinical role of ${ }^{99 \mathrm{~m}} \mathrm{TcO} 4 / \mathrm{MIBI}$ scan, ultrasound, and intraoperative gamma probe in the performance of unilateral and minimally invasive surgery in primary hyperparathyroidism. Eur J Nucl Med 2001;28:1351.

3. Rubello D, Casara D, Pelizzo MR. Symposium on parathyroid localising imaging: Optimization of peroperative procedures. $\mathrm{Nucl}$ Med Commun 2003;24: 133.

4. Rubello D, Casara D, Giannini S, et al. Importance of radioguided minimally invasive parathyroidectomy using hand-held gamma probe and low ${ }^{99 \mathrm{~m}} \mathrm{Tc}-\mathrm{MIBI}$ dose: Technical considerations and long-term clinical results. Q J Nucl Med 2003;47:129.

5. Rubello D, Piotto A, Casara D, et al. Role of gamma probes in performing minimally invasive parathyroidectomy in patients with primary hyperparathyroidism: Optimization of preoperative and intraoperative procedures. Eur J Endocrinol 2003;149:7.

6. Rubello D, Piotto A, Pagetta C, et al. ${ }^{99 \mathrm{~m}}$ Tc-MIBI radioguided surgery for recurrent thyroid carcinoma:
Technical feasibility and procedure, and preliminary clinical results. Eur J Nucl Med 2002;29:1201.

7. Pelizzo MR, Pavan N, Piotto A, et al. Sestamibi radioguided surgery in iodine-131 negative metastatic thyroid cancer. Eur J Nucl Med Mol Imaging 2005;46: P607.

8. Norman J, Chheda H. Minimally invasive parathyroidectomy facilitated by intraoperative nuclear mapping. Surgery 1997;122:998.

9. Murphy C, Norman J. The $20 \%$ rule: A simple, instantaneous radioactivity measurement defines cure and allows elimination of frozen sections and hormone assays during parathyroidectomy. Surgery 1999;126:1023.

10. Costello D, Norman J. Minimally invasive radioguided parathyroidectomy. Surg Oncol Clin N Am 1999;8:555.

11. Flynn MB, Bumpouns JM, Schill K, et al. Minimally invasive radioguided parathyroidectomy. $\underline{\mathrm{J} \mathrm{Am} \text { Coll }}$ Surg 2000;191:24.

12. McGreal G, Winter DC, Sookhai S, et al. Minimally invasive, radioguided surgery for primary hyperparathyroidism. Ann Surg Oncol 2001;8:856.

13. Salvatori M, Rufini V, Reale F, et al. Radioguided surgery for lymph node recurrences of differentiated thyroid cancer. World J Surg 2003;27:770.

14. Rubello D, Saladini G, Casara D, et al. Parathyroid imaging with pertechnetate plus perchlorate/MIBI subtraction scintigraphy: A fast and effective technique. Clin Nucl Med 2000;25:527.

15. Clark OH. Surgical treatment of primary hyperparathyroidism. Adv Endocrinol Metab 1995;6:1.

16. Boz A, Arici C, Güngör F. Gamma probe-guided resection and scanning with Tc-99m MIBI of a local recurrence of follicular thyroid carcinoma. $\mathrm{Clin} \mathrm{Nucl} \mathrm{Med}$ 2001;26:820.

17. Sackett WR, Barraclough B, Reeve TS, et al. Worldwide trends in the surgical treatment of primary hyperparathyroidism in the era of minimally invasive parathyroidectomy. Arch Surg 2002;137:1055.

18. Coakley AJ, Kettle AG, Wells CP, et al. ${ }^{99 \mathrm{~m}} \mathrm{Tc}$-sestamibi-a new agent for parathyroid imaging. $\mathrm{Nucl} \mathrm{Med}$ Commun 1989;10:791.

19. Taillefer R, Boucher Y, Potvin C, et al. Detection and localization of parathyroid adenomas in patients with hyperparathyroidism using a single radionuclide imaging procedure with technetium-99m-Sestamibi (doublephase study). J Nucl Med 1992;33:1801.

20. O'Doherty MJ, Kettle AG, Wells P, et al. Parathyroid imaging with technetium-99m-Sestamibi: Preoperative localization and tissue uptake studies. $\underline{\mathrm{J} \mathrm{Nucl} \mathrm{Med}}$ 1992;33:313.

21. Hindié E, Melliere D, Jeanguillaume C, et al. Parathyroid imaging using simultaneous double-window recording of technetium-99m-sestamibi and iodine-123. J Nucl Med 1998;39:1100.

22. O’Doherty MJ, Kettle AG. Symposium on parathyroid localization. Parathyroid imaging: Preoperative localization. Nucl Med Commun 2003;24:125.

23. Coakley AJ. Symposium on parathyroid localization. [editorial] Nucl Med Commun 2003;24:111. 
24. Mariani G, Gulec SA, Rubello D, et al. Preoperative localization and radioguided parathyroid surgery. $\underline{\mathrm{J} \mathrm{Nucl}}$ Med 2003;44:1443.

25. Sfakianakis GN, Irvin GL, Foss J, et al. Efficient parathyroidectomy guided by SPECT-MIBI and hormonal measurements. J Nucl Med 1996;37:798.

26. Moka D, Voth E, Dietlein M, et al. Technetium 99mMIBI-SPECT: A high sensitive diagnostic tool for localization of parathyroid adenomas. Surgery 2000;128:29.

27. Carty SE, Worsey MJ, Virji MA, et al. Concise parathyroidectomy: The impact of preoperative STECT ${ }^{99 \mathrm{~m}} \mathrm{Tc}$ sestamibi scanning and intraoperative quick parathyroid hormone assay. Surgery 1997;122:1107.

28. Spanu A, Falchi A, Manca A, et al. The usefulness of neck pinhole SPECT as a complementary tool to planar scintigraphy in primary and secondary hyperparathyroidism. J Nucl Med 2004;45:40.

29. Spanu A, Schillaci O, Madeddu G. (99m)Tc-labelled cationic lipophilic complexes in malignant and benign tumors: The role of SPET and pinhole-SPET in breast cancer, differentiated thyroid carcinoma, and hyperparathyroidism. Q J Nucl Med Mol Imaging 2005;49.

30. Gayed IW, Kim EE, Broussard WF, et al. The value of 99mTc-sestamibi SPECT/CT over conventional SPECT in the evaluation of parathyroid adenomas or hyperplasia. J Nucl Med 2005;46:248.

31. Rubello D, Pelizzo MR, Casara D. Nuclear medicine and minimally invasive surgery of parathyroid adenomas: A fair marriage. [editorial] Eur J Nucl Med 2002;30:189.

32. Rubello D, Pelizzo MR, Boni G, et al. Radioguided surgery of primary hyperparathyroidism using the low- dose ${ }^{99 m}$ Tc-sestamibi protocol: Multi-institutional experience from the Italian Study Group on Radioguided Surgery and Immunoscintigraphy (GISCRIS). $\underline{\mathrm{J} \mathrm{Nucl}}$ Med 2005;46:220.

33. Voutilainen PE, Multanen MM, Leppaniemi AK. Prognosis after lymph node recurrence in papillary thyroid carcinoma depends on age. Thyroid 2001;11:953.

34. Robbins RJ, Schlumberger MJ. The evolving role of ${ }^{131} \mathrm{I}$ for the treatment of differentiated thyroid carcinoma. $\underline{J}$ Nucl Med 2005;46:28S.

35. Rubello D, Mazzarotto R, Casara D. The role of technetium-99m methoxyisobutylisonitrile scintigraphy in the planning of therapy and follow-up of patients with differentiated thyroid carcinoma after surgery. Eur J Nucl Med 1999;27:431.

36. Franceschi M, Kusic Z, Franceschi D, et al. Thyroglobulin determination, neck ultrasonography, and iodine-131 whole-body scintigraphy in differentiated thyroid carcinoma. J Nucl Med 1996;37:446.

37. Al-Nahhas A. Dedifferentiated thyroid carcinoma: The imaging role of ${ }^{18} \mathrm{~F}-\mathrm{FDG}-\mathrm{PET}$ and noniodine radiopharmaceuticals. Nucl Med Commun 2004;25:891.

38. Robbins RJ, Wan O, Grewal RK, et al . Real-time prognosis for metastatic thyroid carcinoma based on 2$\left[{ }^{18}\right.$ F]fluoro-2-deoxy-D-glucose-positron emission tomography scanning. J Clin Endocrinol Metab 2006;91:498.

39. Kraeber-Bodere F, Cariou B, Curtet C. et al. Feasibility and benefit of fluorine 18-fluoro-2-deoxyglucoseguided surgery in the management of radioiodine-negative differentiated thyroid carcinoma metastases. Surgery 2005;138:1176. 


\section{This article has been cited by:}

1. P. Paredes, S. Vidal-Sicart, G. Zanón, N. Roé, S. Rubí, S. Lafuente, J. Pavía, F. Pons. 2008. Radioguided occult lesion localisation in breast cancer using an intraoperative portable gamma camera: first results. European Journal of Nuclear Medicine and Molecular Imaging 35:2, 230-235. [CrossRef]

2. Domenico Rubello, Giuliano Mariani. 2007. Hand-held gamma probe or hand-held miniature gamma camera for minimally invasive parathyroidectomy: competition, evolution or synergy?. European Journal of Nuclear Medicine and Molecular Imaging 34:2, 162-164. [CrossRef]

3. Bulent Turgut, Sahande Elagoz, Taner Erselcan, Ayhan Koyuncu, H. Sebila Dokmetas, Zekiye Hasbek, Semra Ozdemir , Cengiz Aydin . 2006. Preoperative Localization of Parathyroid Adenomas with Technetium-99m Methoxyisobutylisonitrile Imaging: Relationship with P-Glycoprotein Expression, Oxyphilic Cell Content, and Tumoral Tissue VolumePreoperative Localization of Parathyroid Adenomas with Technetium-99m Methoxyisobutylisonitrile Imaging: Relationship with P-Glycoprotein Expression, Oxyphilic Cell Content, and Tumoral Tissue Volume. Cancer Biotherapy \& Radiopharmaceuticals 21:6, 579-590. [Abstract] [PDF] [PDF Plus] 